\title{
Diversity of powdery mildew fungi from North Western Himalayan Region of Himachal Pradesh - a checklist
}

\section{Gautam AK ${ }^{1}$ and Avasthi $S^{2}$}

\author{
${ }^{1}$ School of Agriculture, Faculty of Science, Abhilashi University, Mandi, Himachal Pradesh, India \\ ${ }^{2}$ Department of Botany, Abhilashi Post Graduate Institute of Sciences, Mandi, Himachal Pradesh, India
}

Gautam AK, Avasthi S 2018 - Diversity of powdery mildew fungi from North Western Himalayan Region of Himachal Pradesh - a checklist. Plant Pathology \& Quarantine 8(1), 78-99, Doi $10.5943 / \mathrm{ppq} / 8 / 1 / 11$

\begin{abstract}
Powdery mildews are obligate biotropic fungal parasites responsible for disease on a wide range of host plants. They are easily recognizable as white powdery growth on leaves, shoots and sometimes on flowers and even on fruits. This checklist of powdery mildew fungi of Himachal Pradesh, India is based on an exhaustive bibliographic survey of the literature. Fifteen genera (Blumeria, Erysiphe, Euoidium, Golovinomyces, Leveillula, Microsphaera, Neoerysiphe, Oidium, Oidopsis, Phyllactinia, Pleochaeta, Podosphaera, Pseudoidium, Sphaerotheca and Uncinula) and 92 species of powdery mildew have been recorded from the state. About 168 plant species belonging to 122 genera and 49 families are infected by these fungi. Molecular studies of powdery mildew fungi from Himachal Pradesh are needed to revise and to classify these fungi in their correct taxonomic position.
\end{abstract}

Key words - bibliographic survey - Erysiphales - fungal parasites - Himalaya - taxonomy

\section{Introduction}

Powdery mildews are a group of fungal parasites belonging to a single order, Erysiphales. They are obligate biotrophs responsible for many common plant diseases. They are widespread on various hosts including agricultural crops, vegetables, trees, herbs, shrubs, grasses, ornamental plants and weeds. Based on literature, it is estimated that more than 7000 plant host species, throughout the world, are attacked by powdery mildew fungi. However, powdery mildew fungi are more common on cultivated crops than on other plant hosts (Pawar \& Patil 2011). The fungi infect almost every group of plants i.e. from grasses to higher angiosperms (Braun \& Cook 2012).

Powdery mildews are easily recognizable on infected plant parts. The first symptom appears as white powdery spots that may occur on both surfaces of leaves, on shoots and sometimes on flowers and fruits. These spots gradually spread over a large area of the leaves and stems. Disease symptoms usually appear with the onset of summer and begin to disappear during scorching heat and rainy season (Pap et al. 2013).

Powdery mildew fungi grow superficially or epiphytically on plant surfaces. These fungi grow abundantly in dry and cool seasons. Leaves infected with powdery mildew may turn completely yellow, die, and fall off, which may expose fruit to sunburn. On some plants, powdery 
mildew may cause the leaves to twist, buckle, or otherwise distort. The present compilation brings together as much information as possible related to powdery mildews reported from Himachal Pradesh. Hopefully, this checklist will help stimulate further taxonomic research on these fungi, not only in Himachal Pradesh but in other parts of India.

Himachal Pradesh is a hilly state covering an area of 55,673 sq. km and situated in the heart of Himalaya in the northern part of India (Fig. 1). The state extends between 30'22'40" $33^{\prime} 12^{\prime} 40^{\prime \prime}$ North and $75^{\prime} 44^{\prime} 55^{\prime}$ " - 79'04'20" East. It is a mountainous state with elevation ranging from about 350 meters to 7,000 meters above sea level. Climatically the state varies from hot and sub-humid tropical in the southern tracts to cold, alpine and glacial in the northern and eastern mountain ranges with more elevation. This is perhaps one of the reasons for the variable and rich biodiversity in the state. Dense evergreen to deciduous forest covers $66 \%$ of the state. Alpine shrub and meadows are distributed in west and northeast Himalaya with alders, birches, rhododendrons and moist alpine shrubs. Orchards are widely scattered and Himachal is said to be the fruit bowl of India. There are 12 major national parks and sanctuaries to conserve the flora and fauna of the main Himalayan range.

Powdery mildew fungi produce specialized absorption cells (haustoria) which extend into the plant epidermal cells to gain their nutrition. Asexual spores (conidia) are produced either singly or in chains on specialized hyphae (conidiophores). Sexual spores (ascospores) are enclosed in spherical fruiting bodies (chasmothecia). The chasmothecia generally do not have any natural opening, hence, asci and ascospores are released when a crack develops in its outer thick wall. A variety of appendages may occur on the surface of the chasmothecia which are sometimes useful to identify the fungus, at least to genus level. The appendages are thought to help attach the fruiting bodies to the host, particularly to the bark of woody plants, where they overwinter. Besides morphology-based taxonomy, powdery mildew fungi are now going through DNA sequence data, which is not only helping to revise the existence of many fungal species, but to determine their taxonomic position as well.

\section{Materials \& Methods}

\section{Data collection}

The checklist was prepared by using following sources of information:

1. Field surveys carried out from 2012 to 2016 in various localities of lower regions of Himachal Pradesh. Infected leaves were examined primarily with a hand-lens and then with a dissecting microscope for the presence of mildew symptoms. A piece of clear adhesive tape was placed on infected leaves and then stripped off to remove fungal structures. The tape was then placed on a microscope slide with a drop of distilled water. The microscopic observations were carried out for morphological characteristics of mycelia on the host, appressoria, size and shape of conidia, conidiophores and chasmothecia.

2. Exhaustive bibliographic survey of the literature published in various national and international journals, books, and magazines. The literature consulted for present study included several books on Indian fungi including: Erysiphaceae of India (Ahmad et al. 2007), Fungi of India (Butler \& Bisby 1931, Vasudeva 1960, Mukherji \& Juneja 1974, Sarbhoy et al. 1975, 1980, Bilgrami et al. 1991, Jamaluddin et al. 2004), Indian Erysiphaceae (Paul \& Thakur 2006b), Powdery Mildews of India: check list (Hosagoudar \& Agarwal 2009), Taxonomic Manual of the Erysiphales (Powdery Mildews) (Braun \& Cook 2012) and New plant fungal diseases during 21st century in India (Gautam et al. 2012).

In addition, the following papers were consulted (Ahmad et al. 1995, 1998, 2006, Bhardwaj et al. 1985, Bharat 2003, 2006, 2009, 2010, 2013, Bharat \& Bhardwaj 2000, Bharat \& Gupta 2010, Braun \& Paul 2009, Chona et al. 1960, Gautam 2014a, 2014b, Gautam \& Avasthi 2016a, 2016b, 2017, Gill 1968, Gill et al. 1961, Gupta \& Bhardwaj 1998, Gupta et al. 2016, Kala \& Gaur 1983, Kumar et al. 1975, Lall \& Gupta 1965, Paul 1984, 1997, Paul \& Bhardwaj 1982, 1987, 2001, Paul \& Kapoor 1983, 1986, Paul \& Kaul 1988, Paul \& Munjal 1982, Paul \& Pal 1984, 
Paul \& Thakur 2004a, 2004b, 2006a, Sharma et al. 1992, 2017, Singh \& Nagaich 1971, Singh \& Raj 1973, Sohi \& Nayar 1969a, 1969b, Sohi et al. 1966, Soni et al. 1993, Sydow 1938).

The names of some powdery mildew species as reported in the cited publications have been replaced by currently accepted names, according to MycoBank (www.mycobank.org) and Species Fungorum (www.speciesfungorum.org) websites and Braun \& Paul (2009).

\section{Results}

Fifteen genera and 92 species of powdery mildew are known from Himachal Pradesh. These are recorded on about 168 plant host species belonging to 122 genera and 49 families. The species richness of fungi was highest in Erysiphe (33 species) followed by Phyllactinia (17), Podosphaera (9), Microsphaera (8), Golovinomyces (6), Pseudoidium (3), Uncinula (3), Euoidium, Neoerysiphe, Oidopsis and Sphaerotheca (2 each), and Blumeria, Leveillula, Oidium and Pleochaeta (1 each).

Thirty hosts of family Asteraceae were infected with powdery mildew followed by Fabaceae (28), Rosaceae (18), Solanaceae (7), Salicaceae (9), Polygonaceae (8), Moraceae (8), Amaranthaceae (6), Apiaceae (5), Brassicaceae (5), Lamicaeae (5 each), Ranunculaceae, Betulaceae, Poaceae, Juglandaceae, and Plantaginaceae (4 each), Berberidaceae, Boraginaceae, Cannabaceae, Euphorbiaceae, Fagaceae, Linaceae, Rubiaceae and Ulmaceae (3 each), Acanthaceae, Combretaceae, Cucurbitaceae, Malvaceae, Meliaceae and Menispermaceae (2 each), Anacardiaceae, Apocynaceae, Balsaminaceae, Capparidaceae, Caprifoliaceae, Chenopodicaeae, Cleomaceae, Convolvulaceae, Elaeagnaceae, Ericaceae, Geraniaceae, Lauraceae, Lythraceae, Nitrariaceae, Onagraceae, Papaveraceae, Phyllanthaceae, Rutaceae, Sapindaceae and Vitaceae (1 each).

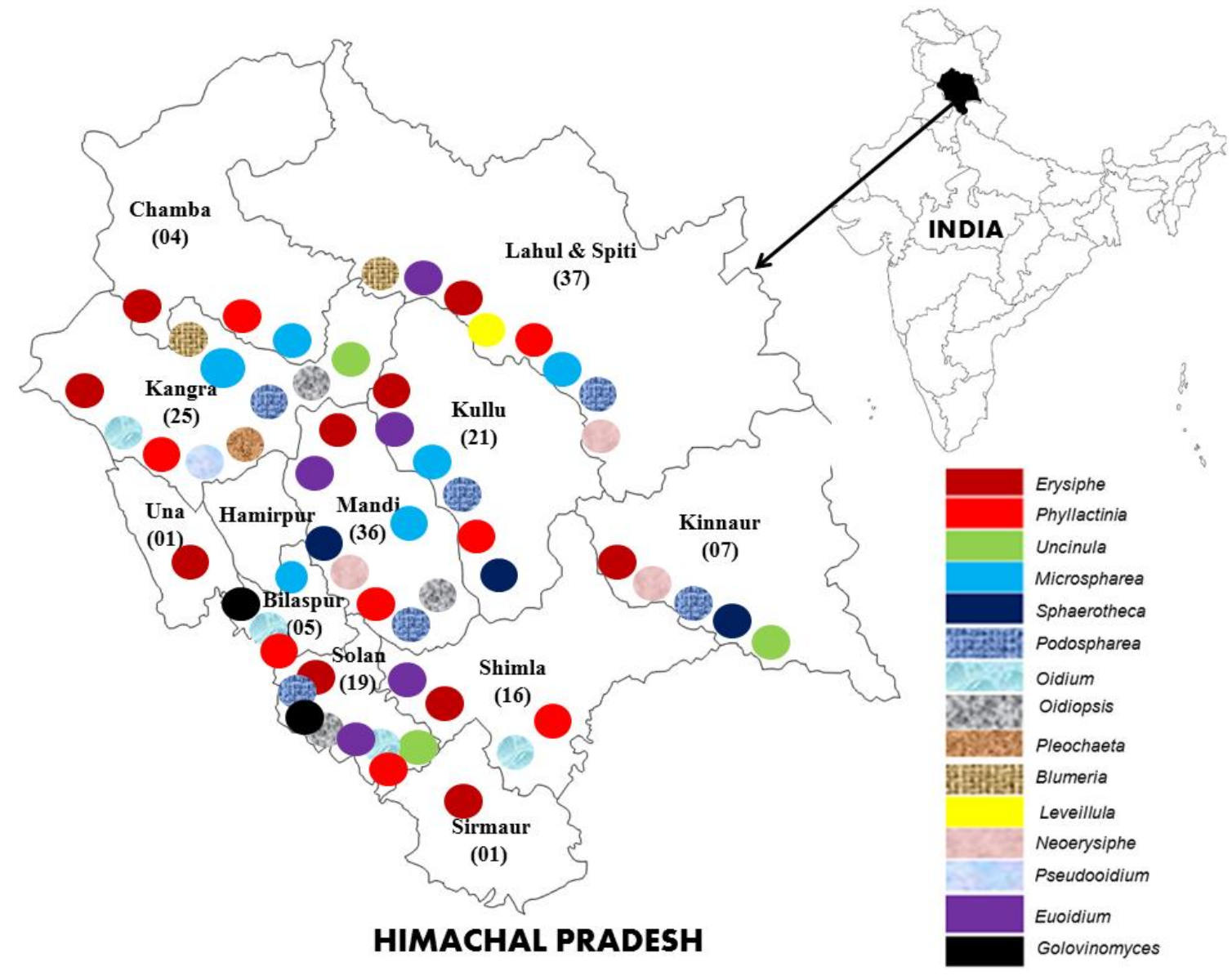

Fig. 1 - Map of study area showing diversity and distribution of powdery mildew fungi in Himachal Pradesh. (Coloured circles illustrate fungal genera and numbers below district name number of records reported from each district). 
A complete list of powdery mildews recorded in Himachal Pradesh, their hosts and distribution is presented in alphabetical order:

Blumeria graminis (DC.) Speer

on Poaceae - Avena fatua, Hordeum vulgare, Triticum aestivum

Distribution - Kukumseri (Lahul \& Spiti), Palampur (Kangra)

Erysiphe adunca (Wallr.) Fr. (syn. Uncinula adunca (Wallr.) Lév.; Uncinula adunca var. regularis (R.Y. Zheng \& G.Q. Chen) U. Braun; Uncinula salicis (DC.) G. Winter) (Fig. 2C)

on Salicaceae - Populus alba, Populus ciliata, Salix sp.

Distribution - Manali (Kullu), Kullu (Kullu), Naggar (Kullu), Shimla (Shimla)

Erysiphe aquilegiae DC. (syn. Erysiphe aquilegiae var. ranunculi (Grev.) R.Y. Zheng \& G.Q. Chen)

on Ranunculaceae - Aconitum heterophyllum

Distribution - Keylong (Lahul \& Spiti)

Erysiphe aquilegiae var. clematidis Y.S. Paul \& V.K. Thakur

on Ranunculaceae - Clematis gauriana

Distribution - Kamrunag (Mandi)

Erysiphe astragali DC. (syn. Microsphaera astragali (DC.) Trevis.)

on Fabaceae - Oxytropis lapponica

Distribution - Moolang (Lahul \& Spiti)

Erysiphe australiana (McAlpine) U. Braun \& S. Takam. (syn. Uncinuliella australiana (McAlpine) R.Y. Zheng \& G.Q. Chen)

on Lythraceae - Lagerstroemia indica

Distribution - Palampur (Kangra)

Erysiphe azalea (U. Braun) U. Braun \& S. Takam. (syn. Erysiphe rhododendri var. barottii Y.S. Paul \& V.K. Thakur)

on Ericaceae - Rhododendron sp.

Distribution - Tikkan (Mandi)

Erysiphe berberidis Y.S. Paul \& J.N. Kapoor

on Berberidaceae - Berberis sp.

Distribution - Chauntra (Mandi)

Erysiphe betae (Vanha) Weltzien (syn. Erysiphe betae var. betae (Vanha) Weltzien; Erysiphe betae var. spinaciarum Y.S. Paul \& J.N. Kapoor) (Fig. 4E)

on Amaranthaceae - Chenopodium ambrosioides, Chenopodium sp., Spinacia oleracea

Distribution - Chauntra (Mandi), Palampur (Kangra), Bajaura (Kullu)

Erysiphe betae var, paulii Y.S. Paul \& J.N. Kapoor

on Amaranthaceae - Beta vulgaris var. benghalensis

Distribution - Palampur (Kangra)

Erysiphe clandestine Biv. (syn. Uncinula clandestina f. clandestine (Biv.) J. Schröt.)

on Betulaceae - Alnus laevigata

Distribution - Dadour (Mandi), Kullu (Kullu) 
Erysiphe clintonii (Peck) U. Braun \& S. Takam. (syn. Uncinula clintonii Peck)

on Moraceae - Morus sp.

Distribution - Kullu (Kullu)

Erysiphe cruciferarum Opiz ex Junell (syn. Erysiphe cruciferarum var. indica Y.S. Paul \& V.K. Thakur)

on Brassicaceae - Coronopus didymus, Lepidium latifolium

Distribution - Baura (Shimla), Tabo (Lahul \& Spiti)

Erysiphe cruciferarum var. chandra Y.S. Paul \& V.K. Thakur

on Brassicaceae - Capsella bursa-pastoris

Distribution - Palampur (Kangra)

Erysiphe cruciferarum var. cleomae Y.S. Paul \& V.K. Thakur

on Cleomaceae - Cleome spinosa

Distribution - Maranda (Kangra)

Erysiphe cummunis (Wallr.) Schltdl.

on Brassicaceae - Lepidium sativum

on Fabaceae - Astragalus sp., Desmodium sp.

Distribution - Dalhausie (Chamba), Kullu (Kullu), Solan (Solan)

Erysiphe ehretiae (Keissl.) U. Braun \& S. Takam. (syn. Uncinula ehretiae Keissl.)

on Boraginaceae - Ehritia acumintata

Distribution - Palampur (Kangra)

Erysiphe farmanii U. Braun \& Y.S. Paul (syn. Farmanomyces sphaerosporus Y.S. Paul \& V.K. Thakur)

on Fagaceae - Quercus glauca

Distribution - Salogra (Solan)

Erysiphe ficicola U. Braun \& Y.S. Paul (syn. Uncinuliella ficicola Y.S. Paul \& V.K. Thakur) on Moraceae - Ficus roxburghii

Distribution - Subathu (Solan)

Erysiphe galii S. Blumer

on Rubiaceae - Rubia sp.

Distribution - Solan (Solan)

Erysiphe heraclei DC. (syn. Erysiphe heraclei var. himalayensis Y.S. Paul \& V.K. Thakur) on Apiaceae - Bunium persicum, Coriandrum sativum, Daucus carota, Selinum veginatum Distribution - Katrain (Kullu), Palampur (Kangra), Sharbo (Kinnaur)

Erysiphe heraclei var. paulii Y.S. Paul \& V.K. Thakur

on Apiaceae - Heracleum candicans

Distribution - Kukumseri (Lahul \& Spiti)

Erysiphe kusanoi (Syd. \& P. Syd.) U. Braun \& S. Takam. (Fig. 2D)

on Cannabaceae - Celtis australis

Distribution - Balt (Mandi) 
Erisiphe lindelophiae Y.S. Paul \& V.K. Thakur

on Boraginaceae - Lindelofia stylosa

Distribution - Molang (Lahul \& Spiti)

Erysiphe machiliana U. Braun \& Y.S. Paul (syn. Uncinuliella machiliana Y.S. Paul \& V.K. Thakur)

on Lauraceae - Machilus odoratissima

Distribution - Saproon (Solan)

Erysiphe malloticola U. Braun \& Y.S. Paul (syn. Uncinula maloti Y.S. Paul \& V.K. Thakur)

on Euphorbiaceae - Mallotus philippensis

Distribution - Kangar (Una), Nahan (Sirmour)

Erysiphe miyabei (E.S. Salmon) U. Braun \& S. Takam. (syn. Uncinula miyabei (E.S. Salmon) Sacc. \& P. Syd.; Uncinula miyabei var. alnicola Y.S. Paul \& V.K. Thakur)

on Betulaceae - Alnus nepalensis, Alnus notidia

Distribution - Kotgarh (Shimla)

Erysiphe mori (Miyake) U. Braun \& S. Takam. (syn. Uncinula mori var. moricola Y.S. Paul \& V.K. Thakur)

on Moraceae - Morus serrata

Distribution - Shimla (Shimla)

Erysiphe munjalii Y.S. Paul \& L.N. Bhardwaj

on Amaranthaceae - Amaranthus mangostans

Distribution: Solan (Solan)

Erysiphe necator Schwein. (syn. Uncinula necator (Schwein.) Burrill)

on Vitaceae - Vitis vinifera

Distribution - Solan (Solan)

Erysiphe paulii U. Braun \& S. Takam. (syn. Uncinula himalayensis Y.S. Paul)

on Solanaceae - Solanum verbascifolium

Distribution - Solan (Solan)

Erysiphe peristrophes (N. Ahmad, A.K. Sarbhoy \& Kamal) U. Braun \& S. Takam. (syn. Uncinula peristrophes N. Ahmad, A.K. Sarbhoy \& Kamal)

on Acanthaceae - Peristrophe bicalyculata

Distribution - Shimla (Shimla)

Erysiphe pirottiana (Bacc.) U. Braun \& S. Takam. (syn. Uncinula pirottiana Bacc.)

on Moraceae - Ficus carica

Distribution - Kotgarh (Shimla)

Erysiphe pisi DC. (syn. Erysiphe pisi var. kukumi Y.S. Paul \& V.K. Thakur)

on Fabaceae - Medicago falcata, Pisum sativum var. arvense, Vicia faba

Distribution - Kukumseri (Lahul \& Spiti), Chauntra (Mandi), Solan (Solan)

Erysiphe pisi var. medicaginis Y.S. Paul \& V.K. Thakur

on Fabaceae - Medicago sativa

Distribution - Kukumseri (Lahul \& Spiti) 
Erysiphe pisi var. pisi DC.

on Fabaceae - Pisum sativum; Vicia faba

Distribution - Kukumseri (Lahul \& Spiti); Tabo (Lahul \& Spiti)

Erysiphe polygoni DC. (syn. Erysiphe polygoni var. rumicis Y.S. Paul \& V.K. Thakur) (Fig. 3B) on Amaranthaceae - Chenopodium ambrosioides

on Polygonaceae - Fagopyrum esculentum, Polygonum aviculare, Rumex patientia

on Ranunculaceae - Clematis gratis

Distribution - Katrain (Kullu), Naggar (Kullu), Chaumtra (Mandi), Tabo (Lahul \& Spiti)

Erysiphe polygoni var.fagopyri Y.S. Paul \& V.K. Thakur

on Polygonaceae - Fagopyrum esculentum

Distribution - Kukumseri (Lahul \& Spiti)

Erysiphe polygoni var. kailashi Y.S. Paul \& V.K. Thakur

on Polygonaceae - Rheum australe

Distribution - Sharbo (Kinnaur)

Erysiphe polygoni var.paulii Y.S. Paul \& V.K. Thakur

on Polygonaceae - Polygonum plebeium

Distribution - Kukumseri (Lahul \& Spiti)

Erysiphe polygoni var. polygoni DC.

on Polygonaceae - Polygonum plebeium

Distribution - Kukumseri (Lahul \& Spiti)

Erysiphe quercicola U. Braun \& Y.S. Paul (Fig. 3D)

on Fagaceae - Quercus incana

Distribution - Balt (Mandi), Chail Chowk (Mandi), Subathu (Solan)

Erysiphe quercifolia U. Braun \& Y.S. Paul (syn. Uncinula quercifolia Y.S. Paul \& V.K. Thakur) on Fagaceae - Quercus incona

Distribution - Subathu (Solan)

Erysiphe religiosa (T.S. Ramakr.) U. Braun \& Y.S. Paul (syn. Uncinula aspera var. solani Y.S. Paul \& V.K. Thakur)

on Moraceae - Ficus religiosa

Distribution - Kotgarh (Shimla), Subathu (Solan)

Erysiphe sordida var. spitiana Y.S. Paul \& V.K. Thakur

on Plantaginaceae - Plantago tabetica

Distribution - Tabo (Lahul \& Spiti)

Erysiphe trifolii Grev. (syn. Erysiphe martii Lév.) (Fig. 2B)

on Fabaceae - Trifolium repens

Distribution - Balt (Mandi), Chail Chowk (Mandi), Palampur (Kangra)

Erysiphe verniciferae (Henn.) U. Braun \& S. Takam. (syn. Uncinula verniciferae Henn.)

on Anacardiaceae - Pistacia integerrima

Distribution - Kotgarh (Shimla) 
Euoidium agerati (J.M. Yen) U. Braun \& R.T.A. Cook (syn. Oidium agerati J.M. Yen) on Asteraceae - Ageratum conyzoides

Distribution - Berthin (Bilaspur)

Euoidium strobilanthis Y.S. Paul \& J.L. Kaul

on Acanthaceae - Strobilanthes sp.

Distribution - Solan (Solan)

Golovinomyces artemisiae (Grev.) V.P. Heluta (syn. Erysiphe artemisiae Grev.)

on Asteraceae - Artemisia scoparia.

Distribution - Shimla (Shimla).

Golovinomyces biocellatus (Ehrenb.) V.P. Heluta (syn. Erysiphe biocellata Ehrenb.)

on Lamiaceae - Mentha sp., Nepeta laevigata

Distribution - Keylong (Lahul \& Spiti), Kukumseri (Lahul \& Spiti)

Golovinomyces cichoracearum (DC.) V.P. Heluta (syn. Erysiphe cichoracearum DC.; Erysiphe cichoracearum var. saussureae Y.S. Paul \& V.K. Thakur) (Figs 3G, 4G)

on Asteraceae - Artemisia maritime, Chrysanthemum sp., Cousinia thomsonii, Dahlia variabilis, Heliantus annuum, Lactuca decipiens, Matricaria chamomilla, Saussurea costus, Sonchus sp.

on Boraginaceae - Eritrichium rupestre

on Brassicaceae - Capsella bursa-pastoris

on Cucurbitaceae - Cucurbita pepo

on Malvaceae - Abelmoschus esculentus

on Plantaginaceae - Veronica biloba

on Rosaceae - Malus sylvestris

Distribution - Baura (Shimla), Chauntra (Mandi), Karpat (Lahul \& Spiti), Kullu (Kullu), Madgran (Lahul \& Spiti), Manali (Kullu), Moorang (Lahul \& Spiti), Nauni (Solan), Solan (Solan), Spiti (Lahul \& Spiti)

Golovinomyces depressus (Wallr.) V.P. Heluta (syn. Erysiphe depressa (Wallr.) Link)

on Asteraceae - Arctium lappa

Distribution - Kukumseri (Lahul \& Spiti)

Golovinomyces orontii (Castagne) V.P. Heluta (syn. Erysiphe orontii var. papaveris Y.S. Paul \& V.K. Thakur; Oidium lini Škorič)

on Linaceae - Linum usitatissimum

on Papaveraceae - Papaver somniferum

Distribution - Kukumseri (Lahul \& Spiti), Shimla (Shimla)

Golovinomyces sordidus (L. Junell) Heluta (syn. Erysiphe sordida var. similensis Y.S. Paul \& V.K. Thakur)

on Plantaginaceae - Plantago major

Distribution - Kukumseri (Lahul \& Spiti)

Leveillula taurica (Lév.) G. Arnaud (syn. Erysiphe lindelofiae Y.S. Paul \& V.K. Thakur)

on Asteraceae - Sonchus asper

on Boraginaceae - Lindelofia stylosa

on Capparidaceae-Capparis spinosa

on Convolvulaceae - Convolvulus arvensis

on Plantaginaceae - Plantago major

Distribution - Spiti Valley (Lahul \& Spiti), Moolang (Lahaul \& Spiti) 
Microsphaera acaciae (S. Blumer) U. Braun

on Fabaceae - Acacia catechu

Distribution - Panjgrain, Barmana (Bilaspur)

Microsphaera barberidis (DC.) Lév.

on Berbidaceae - Barberis vulgaris

Distribution - Kukumseri (Lahul \& Spiti)

Microsphaera caprifoliacearum var. caprifoliacearum U. Braun

on Caprifoliaceae - Lonicera sp.

Distribution - Rohanda (Mandi)

Microsphaera hydsari Braun

on Fabaceae - Hedysarum cachumarianum

Distribution - Kayardo (Lahul \& Spiti)

Microsphaera juglendis var. paulii Y.S. Paul \& V.K. Thakur

on Juglandaceae - Juglens regia

Distribution - Bharmour (Chamba)

Microsphaera martii (Lév.) Y.S. Paul \& V.K. Thakur

on Fabaceae - Trigonella foenum-graecum

Distribution - Palampur (Kangra)

Microsphaera prasadii M.K. Bhatn. \& K.L. Kothari

on Rubiaceae - Hamiltonia suaveolens

Distribution - Ner Chowk (Mandi)

Microsphaera trifolii (Grev.) U. Braun

on Fabaceae - Trifolium repens

Distribution - Diphu, Katrain (Kullu)

Neoerysiphe galeopsidis (DC.) U. Braun (syn. Arthrocladiella althaeae Y.S. Paul \& V.K. Thakur; Erysiphe galeopsidis DC.)

on Lamiaceae - Phlomis bracteosa

on Malvaceae - Althaea rosea

Distribution - Sharbo (Kinnaur), Chhatru (Lahul \& Spiti).

Neoerysiphe geranii (Y. Nomura) U. Braun (syn. Erysiphe kapoorii Y.S. Paul \& V.K. Thakur) on Geraniaceae - Geranium wallichianum

Distribution - Karsog (Mandi)

Oidiopsis solani N. Ahmad, A.K. Sarbhoy, Kamal \& D.K. Agarwal on Nitrariaceae - Peganum harmala

on Solanaceae - Solanum melongena

Distribution - Jai Singhpur (Kangra)

Oidiopsis taurica (Lév.) E.S. Salmon

on Linaceae - Reinwardtia indica

Distribution - Palampur (Kangra) 
Oidiopsis taurica f.sp. balsaminacearum Y.S. Paul \& J.N. Kapoor (Fig. 3A)

On Balsaminaceae - Impatiens balsamina

Distribution - Palampur (Kangra)

Oidiopsis taurica f.sp. euphorbiacearum Y.S. Paul \& J.N. Kapoor

on Euphorbiaceae - Euphorbia geniculata

Distribution - Palampur (Kangra)

Oidiopsis taurica f.sp. ranunculacearum Y.S. Paul \& J.N. Kapoor

on Ranunculaceae - Delphinium ajacis

Distribution - Kasauli (Solan)

Oidiopsis taurica f.sp. rosacearum Y.S. Paul \& J.N. Kapoor

on Rosaceae - Amygdalus communis

on Solanaceae - Solanum melongena

Distribution - Chauntra (Mandi)

Oidiopsis taurica f.sp. solonacearum Y.S. Paul \& J.N. Kapoor

on Solanaceae - Capsicum annum, Capsicum frutescens, Solanum melongena, Solanum tuberosum Distribution - Chauntra (Mandi)

Oidium sp. (Fig. 3F)

on Amarantaceae - Amaranthus sp.

on Asteraceae - Coreopsis sp., Eupatorium cannabinum, Galinsoga parviflora, Vernonia cinerea

on Fabaceae - Lathyrus sp.

on Lamiaceae - Coleus barbatus, Nepeta leucophylla

on Menispermaceae - Stephania rotunda

on Rutaceae - Aegle marmelos

on Solanaceae - Datura stramonium, Nicandra physalodes, Petunia sp.

on Urticaceae - Urtica dioica

Distribution - Shimla (Shimla), Palampur (Kangra), Solan (Solan), Berthin (Bilaspur)

Phyllactinia alnicola U. Braun (syn. Microsphaera alni (DC.) G. Winter)

on Juglandaceae - Juglans regia

on Salicaceae - Flacourtia sapida

Distribution - Kullu (Kullu)

Phyllactinia andrachnes H.S. Gill, Munjal \& Chona

on Phyllanthaceae - Andrachne cordifolia

Distribution - Kullu (Kullu)

Phyllactinia bauhiniae Y.S. Paul (syn. Phyllactinia bauhiniae Y.S. Paul)

on Fabaceae - Bauhinia sp.

Distribution - Samela (Kangra)

Phyllactinia belliricae var. cedrelae Y.S. Paul \& V.K. Thakur

on Combretaceae - Terminalia bellirica

Distribution - Palampur (Kangra)

Phyllactinia cassiae-fistulae U. Braun \& Y.S. Paul (syn. Phyllactinia bauhiniae var. cassia Y.S. Paul \& V.K. Thakur)

on Fabaceae - Cassia fistula 
Distribution - Panjgrain, Barmana (Bilaspur)

Phyllactinia delbergiae Piroz.

on Fabaceae - Dalbergia sissoo

Distribution - Panjgrain, Barmana (Bilaspur)

Phyllactinia guttata (Wallr.) Lév. (syn. Phyllactinia corylea (Pers.) P. Karst; Phyllactinia suffulta (Rebent.) Sacc.; Phyllactinia guttata var. guttata (Wallr.) Lév.) (Fig. 2E)

on Berberidaceae - Barberis lycium

on Betulaceae - Alnus nitida

on Fabaceae - Caesalpinia sp., Desmodium sp., Robinia pseudoacacia

on Meliaceae - Cedrela toona, Toona ciliata

on Moraceae - Morus alba, Morus serrata, Morus sp.

on Rosaceae - Cotoneaster bacillaris, Cydonia oblonga, Prunus sp., Pyrus serotina, Spiraea sp.

on Salicaceae - Salix babylonica, Salix sp.

on Ulmaceae - Ulmus laevigata

Distribution - Balt (Mandi), Bharmour (Chamba), Chail Chowk (Mandi), Dalhausie (Chamba), Jhantigri (Mandi), Jogindernagar (Mandi), Kullu (Kullu), Manali (Kullu), Palampur (Kangra), Solan (Solan)

Phyllactinia hippophaes Thüm. ex S. Blumer

on Elaeagnaceae - Hippophae sp.

Distribution - Kukumseri (Lahul and Spiti)

Phyllactinia juglandis var.junglandae Y.S. Paul \& V.K. Thakur

on Junglandaceae - Juglans regia

Distribution - Karsog (Mandi)

Phyllactinia juglandis var. paulii Y.S. Paul \& V.K. Thakur (Fig. 4F)

on Junglandaceae - Junglans regia

Distribution - Karsog (Mandi)

Phyllactinia mali (Duby) U. Braun (Fig. 4D)

on Rosaceae - Pyrus communis; Pyrus pashia

Distribution - Naggar (Kullu), Shimla (Shimla)

Phyllactinia miracula Y.S. Paul

on Elaeagnaceae - Hippophae sp.

Distribution - Lahul Valley (Lahul \& Spiti)

Phyllactinia populi (Jacz.) Y.N. Yu. (syn. Phyllactinia guttata var. populina Y.S. Paul \& V.K. Thakur) (Fig. 2E)

on Saliciaceae - Populus sp.

Distribution - Karsog (Mandi)

Phyllactinia pyri-serotinae Sawada (syn. Phyllactinia guttata var. rosacearum Y.S. Paul \& V.K.

Thakur)

on Rosaceae - Cotonearter bacillaris

on Salicaceae - Populus sp.

Distribution - Shimla (Shimla), Solan (Solan) 
Phyllactinia roboris (Gachet) S. Blumer

on Solanaceae - Solanum verbascifolium

Distribution - Solan (Kullu)

Phyllactinia terminaliae T.S. Ramakr (syn. Phyllactinia belliricae Y.S. Paul \& V.K. Thakur)

on Combretaceae - Terminalia bellirica

Distribution - Palampur (Kangra)

Phyllactinia toonae Y.X. Yu \& Y.Q. Lai. (syn. Phyllactinia guttata var. cedrelae Y.S. Paul \& V.K. Thakur)

on Meliaceae - Cedrela toona

Distribution - Palampur (Kangra)

Pleochaeta shiraiana (Henn.) Kimbr. \& Korf.

on Cannabaceae - Celtis australis

Distribution - Palampur (Kangra)

Podosphaera ainsliaeae (Y.S. Paul \& V.K. Thakur) U. Braun \& Y.S. Paul (syn. Sphaerotheca ainsliaeae Y.S. Paul \& V.K. Thakur)

on Asteraceae - Ainsliaea aptera

Distribution - Karsog (Mandi)

Podosphaera aphanis (Wallr.) U. Braun \& S. Takam. (syn. Sphaerotheca aphanis var. potentillae Y.S. Paul \& V.K. Thakur)

on Rosaceae - Potentilla sp.

Distribution - Hansa (Lahul \& Spiti)

Podosphaera clandestina (Wallr.) Lév. (syn. Podosphaera clandestina var. cydoniae N. Ahmad, A.K. Sarbhoy \& Kamal)

on Rosaceae - Cydonia oblonga

Distribution - Kullu (Kullu)

Podosphaera euphorbiae-hirtae (U. Braun \& Somani) U. Braun \& S. Takam (Fig. 4B)

on Euphorbiaceae - Euphorbia hirta

Distribution - Balt (Mandi), Chail Chowk (Mandi)

Podosphaera fuliginea (Schltdl.) U. Braun \& S. Takam. (syn. Oidium erysiphoides Fr.; Sphaerotheca fuliginea (Schltdl.) Pollacci) (Fig. 3C)

on Apiaceae - Heracleum candicans

on Asteraceae - Calendula sp., Conyza stricta, Cosmos sp., Erigeron bonariensis, Zinnia elegans

on Cucurbitaceae - Cucurbita moschata

on Fabaceae - Dolichos biflorus, Vigna mungo, Vigna radiata, Vigna umbellata

on Linaceae - Macrolium sp.

on Rubiaceae - Galium aparine

Distribution - Balt (Mandi), Chail Chowk (Mandi), Kullu (Kullu), Palampur (Kangra), Solan (Solan)

Podosphaera fusca (Fr.) U. Braun \& Shishkoff (syn. Podosphaera xanthii (Castagne) U. Braun \& Shishkoff; Sphaerotheca ainsliaeae Y.S. Paul \& V.K. Thakur; Sphaerotheca fusca (Fr.) S. Blumer; Sphaerotheca fusca var. compositarum Y.S. Paul \& V.K. Thakur; Sphaerotheca phaseoli (Z.Y. Zhao) U. Braun) (Figs 2A, 3E, 4C) 
on Asteraceae - Ageratum conyzoides, Ainsliaea aptera, Blumea sp., Calendula sp., Coreopsis lanceolata; Senecio chrysanthemoides, Sonchus sp., Taraxcum officinale, Xanthium strumarium on Fabaceae - Phaseolus mungo

Distribution - Balt, Chail Chowk (Mandi), Chhatru, Keylong, Udaipur, Kukumseri (Lahul \& Spiti), Jachh, Karsog (Mandi)

Podosphaera leucotricha (Ellis \& Everh.) E.S. Salmon

on Rosaceae - Malus domestica

Distribution - Kalpa (Kinnaur), Sharbo (Kinnaur)

Podosphaera tridactyla (Wallr.) de Bary (syn. Podosphaera tridactyla var. prunicola Y.S. Paul \& V.K. Thakur; Podosphaera tridactyla var. tridactyla (Wallr.) de Bary)

on Rosaceae - Prunus cornuta

Distribution - Madgran (Lahul \& Spiti)

Podosphaera xanthii (Castagne) U. Braun \& Shishkoff (syn. Sphaerotheca fusca var. cucumis Y.S. Paul \& V.K. Thakur; Sphaerotheca heteropogonis Y.S. Paul \& V.K. Thakur)

on Poaceae - Heteropogon contortus

Distribution - Padar (Mandi)

Pseudoidium cryptolepidis (Hosag., Vijay., Udaiyan \& Siddappa) U. Braun \& R.T.A. Cook on Apocynaceae - Cryptolepis buchanani

Distribution - Balt (Mandi), Chail Chowk (Mandi)

Pseudoidium indica Y.S. Paul \& L.N. Bhardwaj

on Onagraceae - Oenothera rosea

Distribution - Palampur (Kangra)

Pseudoidium prinsepiae (Y.S. Paul \& L.N. Bhardwaj) U. Braun \& R.T.A. Cook (syn. Oidium prinsepiae Y.S. Paul \& L.N. Bhardwaj)

on Rosaceae - Prinsepia utilis

Distribution - Mandi (Mandi)

Sphaerotheca fagopyri Syd. \& Butler

on Polygonaceae - Fagopyrum esculentum

Distribution - Kullu (Kullu)

Sphaerotheca fuliginea var. galinsogae Y.S. Paul \& J. Paul

on Asteraceae - Galinsoga parviflora

Distribution - Sharbo (Kinnaur)

Uncinula aceris (DC.) Sacc.

on Sapindaceae - Acer oblongum

Distribution - Solan (Solan)

Uncinula aspera var. aspera U. Braun

on Moraceae - Ficus carica

Distribution - Sharbo (Kinnaur)

Uncinula celtidis var. celtidis Y.S. Paul \& V.K. Thakur

on Cannabaceae - Celtis australis

Distribution - Palampur (Kangra) 


\section{Host index}

In this section scientific name of host plants along with the powdery mildew fungi occurring on them are provided.

Abelmoschus esculentus - Golovinomyces cichoracearum

Acacia catechu - Microsphaera acacia

Acer oblongum - Uncinula aceris

Aconitum heterophyllum - Erysiphe aquilegiae

Aegle marmelos - Oidium sp.

Ageratum conyzoides - Euoidium agerati, Podosphaera fuliginea

Ainsliaea aptera - Podosphaera ainsliaeae, Podosphaera fuliginea

Alnus laevigata - Erysiphe clandestina

Alnus nepalensis - Erysiphe miyabei

Alnus nitida - Phyllactinia guttata

Alnus notidia - Erysiphe miyabei

Althaea rosea - Neoerysiphe galeopsidis

Amaranthus mangostans - Erysiphe munjalii

Amaranthus sp. - Oidium sp.

Amygdalus communis - Oidiopsis taurica f.sp. rosacearum

Andrachne cordifolia - Phyllactinia andrachnes

Arctium lappa - Golovinomyces depressus

Artemisia maritime - Golovinomyces cichoracearum

Artemisia scoparia. - Golovinomyces artemisiae

Astragalus sp. - Erysiphe cummunis

Avena fatua - Blumeria graminis

Barberis lycium - Phyllactinia guttata

Barberis vulgaris - Microsphaera barberidis

Bauhinia sp. - Phyllactinia bauhiniae

Berberis sp. - Erysiphe berberidis

Beta vulgaris - Erysiphe betae var. paulii

Blumea sp. - Podosphaera fuliginea

Bunium persicum - Erysiphe heraclei

Caesalpinia sp. - Phyllactinia guttata

Calendula sp. - Podosphaera fuliginea

Capparis spinosa-Leveillula taurica

Capsella bursa-pastoris - Erysiphe cruciferarum var. chandra, Golovinomyces cichoracearum

Capsicum annum - Oidiopsis taurica f.sp. solonacearum

Capsicum frutescens - Oidiopsis taurica f.sp. solonacearum

Cassia fistula - Phyllactinia cassiae-fistulae

Cedrela toona - Phyllactinia guttata, Phyllactinia toonae

Celtis australis - Erysiphe kusanoi, Pleochaeta shiraiana, Uncinula celtidis var. celtidis

Chenopodium ambrosioides - Erysiphe betae, Erysiphe polygoni

Chenopodium sp. - Erysiphe betae

Chrysanthemum sp. - Golovinomyces cichoracearum

Clematis gauriana - Erysiphe aquilegiae var. clematidis

Clematis gratis - Erysiphe polygoni

Cleome spinosa-Erysiphe cruciferarum var. cleomae

Coleus barbatus - Oidium sp.

Convolvulus arvensis - Leveillula taurica

Conyza stricta - Podosphaera fuliginea

Coreopsis lanceolata-Podosphaera fuliginea

Coreopsis sp. - Oidium sp. 
Coriandrum sativum - Erysiphe heraclei

Coronopus didymus - Erysiphe cruciferarum

Cosmos sp. - Podosphaera fuliginea

Cotonearter bacillaris - Phyllactinia guttata, Phyllactinia pyri-serotinae

Cousinia thomsonii - Golovinomyces cichoracearum

Cryptolepis buchanani - Pseudoidium cryptolepidis

Cucurbita moschata-Podosphaera fuliginea

Cucurbita pepo-Golovinomyces cichoracearum

Cydonia oblonga - Phyllactinia guttata, Podosphaera clandestina

Dahlia variabilis - Golovinomyces cichoracearum

Dalbergia sissoo - Phyllactinia delbergiae

Datura stramonium - Oidium sp.

Daucus carota - Erysiphe heraclei

Delphinium ajacis - Oidiopsis taurica f.sp. ranunculacearum

Desmodium sp. - Erysiphe cummunis, Phyllactinia guttata

Dolichos biflorus - Podosphaera fuliginea

Ehritia acumintata - Erysiphe ehretiae

Erigeron bonariensis - Podosphaera fuliginea

Eritrichium rupestre - Golovinomyces cichoracearum

Eupatorium cannabinum - Oidium sp.

Euphorbia geniculate - Oidiopsis taurica f.sp. euphorbiacearum

Euphorbia hirta-Podosphaera euphorbiae-hirtae

Fagopyrum esculentum - Erysiphe polygoni, Erysiphe polygoni var. fagopyri, Sphaerotheca fagopyri

Ficus carica - Erysiphe pirottiana, Uncinula aspera var. aspera

Ficus religiosa - Erysiphe religiosa

Ficus roxburghii - Erysiphe ficicola

Flacourtia sapida - Phyllactinia alnicola

Galinsoga parviflora-Oidium sp., Sphaerotheca fuliginea var. galinsogae

Galium aparine - Podosphaera fuliginea

Geranium wallichianum - Neoerysiphe geranii

Hamiltonia suaveolens - Microsphaera prasadii

Hedysarum cachumarianum - Microsphaera hydsari

Heliantus annuum - Golovinomyces cichoracearum

Heracleum candicans - Erysiphe heraclei var. paulii, Podosphaera fuliginea

Heteropogon contortus - Podosphaera xanthii

Hippophae sp. - Phyllactinia hippophaes, Phyllactinia miracula

Hordeum vulgare - Blumeria graminis

Impatiens balsamina - Oidiopsis taurica f.sp. balsaminacearum

Juglans regia - Microsphaera juglandis var. paulii, Phyllactinia alnicola, Phyllactinia juglandis

var. junglandae, Phyllactinia juglandis var. paulii

Lactuca decipiens - Golovinomyces cichoracearum

Lagerstroemia indica - Erysiphe australiana

Lathyrus sp. - Oidium sp.

Lepidium latifolium - Erysiphe cruciferarum

Lepidium sativum - Erysiphe cummunis

Lindelofia stylosa - Erysiphe lindelophiae, Leveillula taurica

Linum usitatissimum - Golovinomyces orontii

Lonicera sp. - Microsphaera caprifoliacearum var. caprifoliacearum

Machilus odoratissima - Erysiphe machiliana

Macrolium sp. - Podosphaera fuliginea

Mallotus philippensis - Erysiphe malloticola 
Malus domestica - Podosphaera leucotricha

Malus sylvestris - Golovinomyces cichoracearum

Matricaria chamomilla - Golovinomyces cichoracearum

Medicago falcata-Erysiphe pisi

Medicago sativa-Erysiphe pisi var. medicaginis

Mentha sp. - Golovinomyces biocellatus

Morus alba - Phyllactinia guttata

Morus serrata - Erysiphe mori, Phyllactinia guttata

Morus sp. - Erysiphe clintonii, Phyllactinia guttata

Nepeta laevigata - Golovinomyces biocellatus

Nepeta leucophylla - Oidium sp.

Nicandra physalodes - Oidium sp.

Oenothera rosea - Pseudoidium indica

Oxytropis lapponica - Erysiphe astragali

Papaver somniferum - Golovinomyces orontii

Peganum harmala - Oidiopsis solani

Peristrophe bicalyculata - Erysiphe peristrophes

Petunia sp. - Oidium sp.

Phaseolus mungo - Podosphaera fuliginea

Phlomis bracteosa - Neoerysiphe galeopsidis

Pistacia integerrima - Erysiphe verniciferae

Pisum sativum - Erysiphe pisi, Erysiphe pisi var. pisi

Plantago major - Golovinomyces sordidus, Leveillula taurica

Plantago tabetica - Erysiphe sordida var. spitiana

Polygonum aviculare - Erysiphe polygoni

Polygonum plebeium - Erysiphe polygoni var. paulii, Erysiphe polygoni var. polygoni

Populus alba - Erysiphe adunca

Populus ciliata - Erysiphe adunca

Populus sp. - Phyllactinia populi, Phyllactinia pyri-serotinae

Potentilla sp. - Podosphaera aphanis

Prinsepia utilis - Pseudoidium prinsepiae

Prunus cornuta - Podosphaera tridactyla

Prunus sp. - Phyllactinia guttata

Pyrus communis - Phyllactinia mali

Pyrus pashia - Phyllactinia mali

Pyrus serotina - Phyllactinia guttata

Quercus glauca-Erysiphe farmanii

Quercus incana - Erysiphe quercicola

Reinwardtia indica-Oidiopsis taurica

Rheum australe - Erysiphe polygoni var. kailashi

Rhododendron sp. - Erysiphe azalea

Robinia pseudoacacia - Phyllactinia guttata

Rubia sp. - Erysiphe galii

Rumex patientia - Erysiphe polygoni

Salix babylonica - Phyllactinia guttata

Salix sp. - Erysiphe adunca, Phyllactinia guttata

Saussurea costus - Golovinomyces cichoracearum

Selinum veginatum - Erysiphe heraclei

Senecio chrysanthemoides - Podosphaera fuliginea

Solanum melongena - Oidiopsis solani, Oidiopsis taurica f.sp. rosacearum, Oidiopsis taurica f.sp. solonacearum

Solanum tuberosum - Oidiopsis taurica f.sp. solonacearum 
Solanum verbascifolium - Erysiphe paulii, Phyllactinia roboris

Sonchus asper - Leveillula taurica

Sonchus sp. - Golovinomyces cichoracearum, Podosphaera fuliginea

Spinacia oleracea - Erysiphe betae

Spiraea sp. - Phyllactinia guttata

Stephania rotunda - Oidium sp.

Strobilanthes sp. - Euoidium strobilanthis

Taraxcum officinale - Podosphaera fuliginea

Terminalia bellirica - Phyllactinia belliricae, Phyllactinia terminaliae

Toona ciliata - Phyllactinia guttata

Trifolium repens - Erysiphe trifolii, Microsphaera trifolii

Trigonella foenum-graecum - Microsphaera martii

Triticum aestivum - Blumeria graminis

Ulmus laevigata - Phyllactinia guttata

Urtica dioica - Oidium sp.

Vernonia cinerea-Oidium sp.

Veronica biloba - Golovinomyces cichoracearum

Vicia faba-Erysiphe pisi, Erysiphe pisi var. pisi

Vigna mungo - Podosphaera fuliginea

Vigna radiata - Podosphaera fuliginea

Vigna umbellata umbellata - Podosphaera fuliginea

Vitis vinifera - Erysiphe necator

Xanthium strumarium - Podosphaera fuliginea

Zinnia elegans - Podosphaera fuliginea
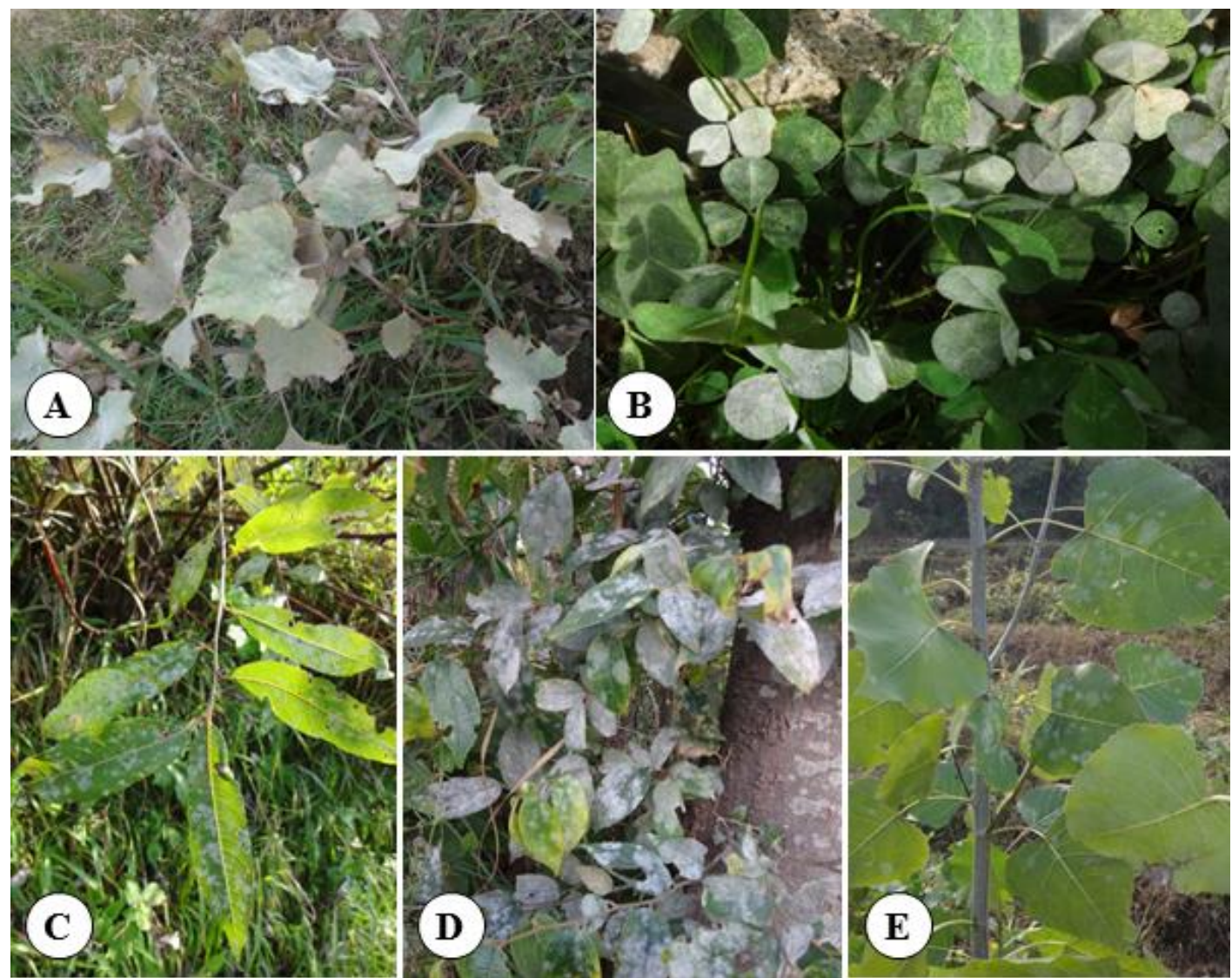

Fig. 2 - Powdery mildews A Podosphaera fusca on Xanthium strumarium. B Erysiphe trifolii on Trifolium repentis. C Erysiphe adunca on Salix sp. D Erysiphe kusanoi on Celtis australis. E Phyllactinia populi on Populus sp. 

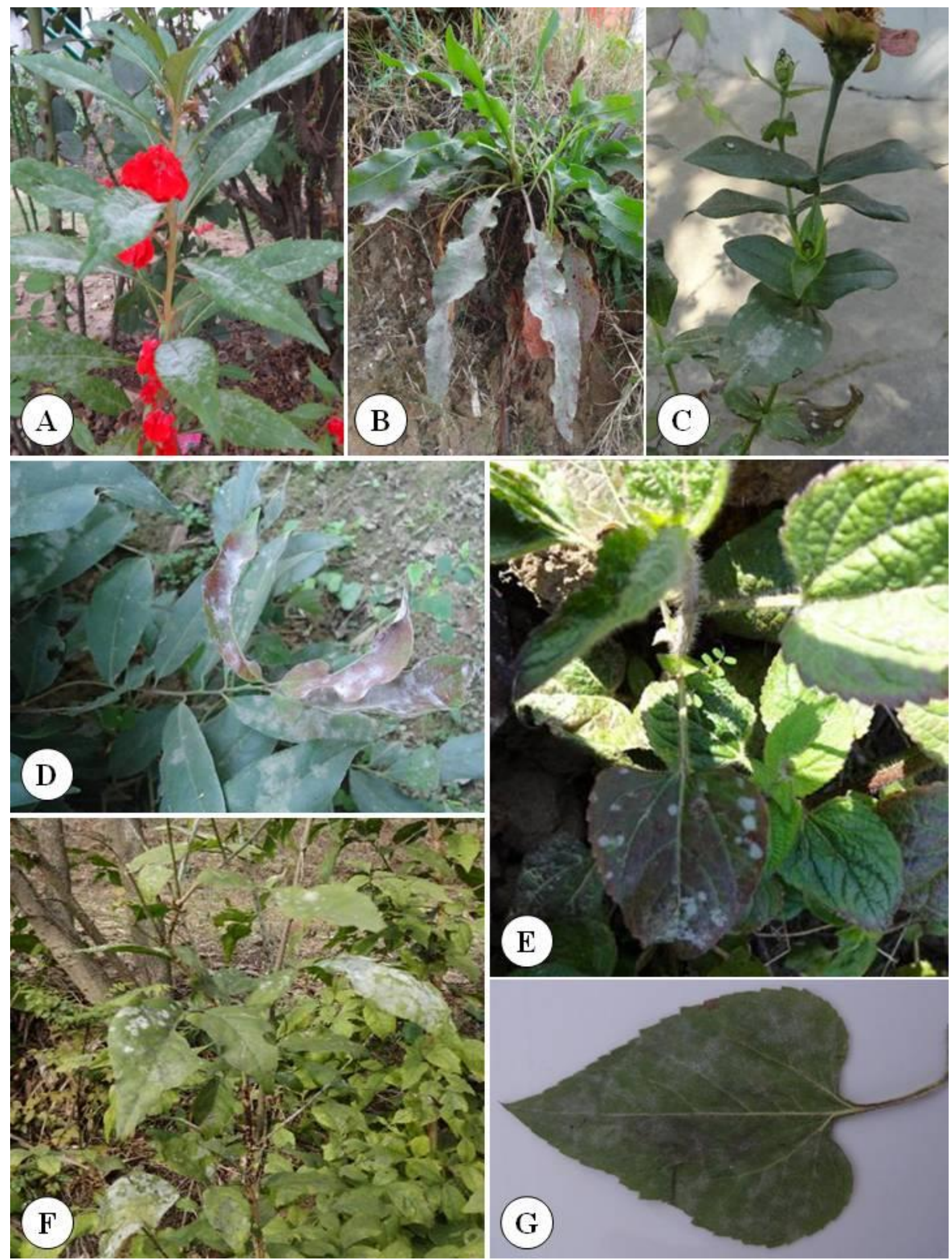

Fig. 3 - Powdery mildews A Odiopsis taurica on Balsam sp. B Erysiphe polygoni on Rumex sp. C Podosphaera fuliginea on Zinnia elegans. D Erysiphe quercicola on Quercus sp. E Podosphaera fusca on Ageratum conyzoides. F Oidium sp. on Aegle marmelos. G Golovinomyces cichoracearum on Helianthus annuus. 


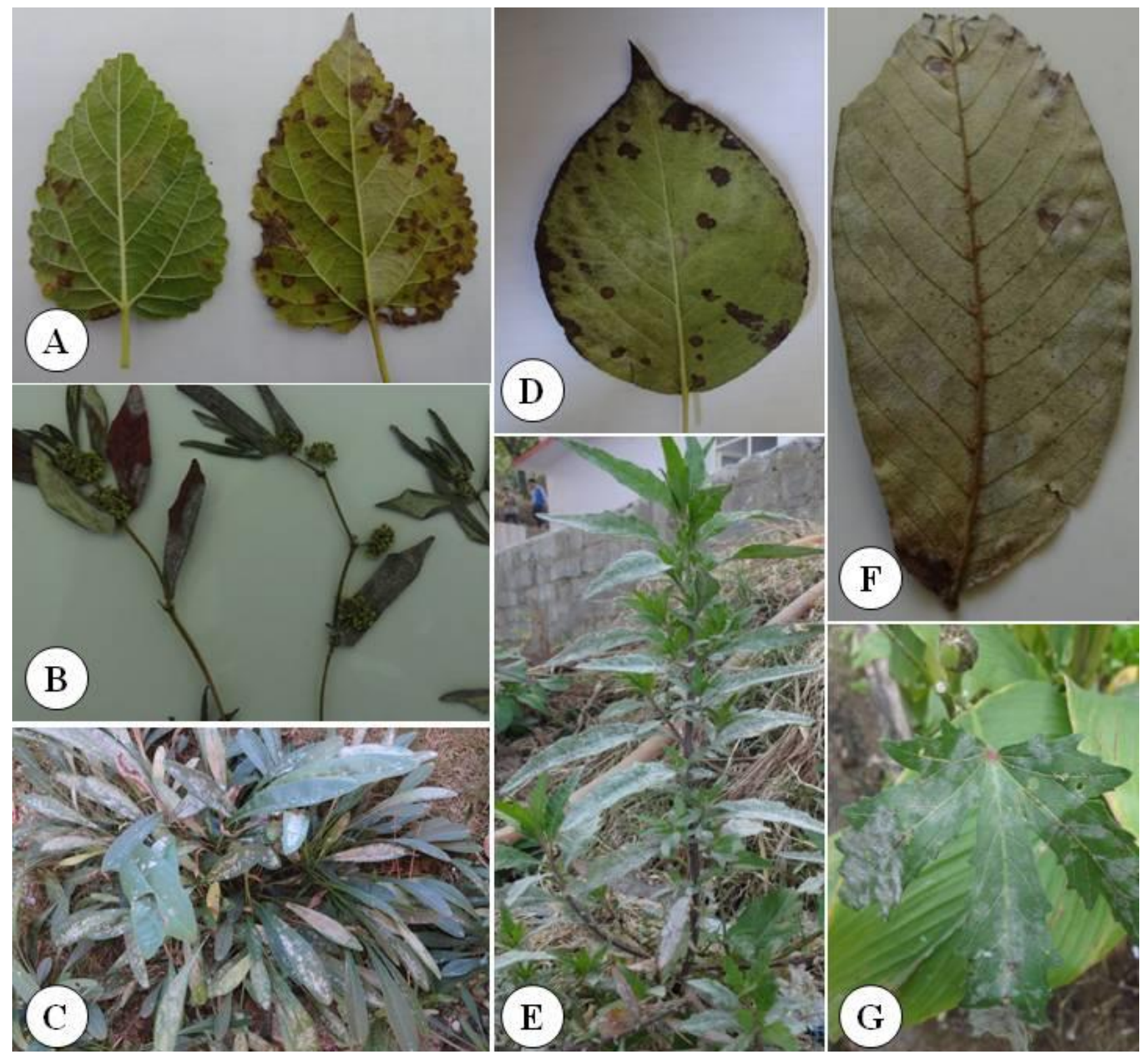

Fig. 4 - Powdery mildews A Phyllactinia guttata on Morus alba. B Podosphaera euphorbiaehirtae on Euphorbia hirta. C Podosphaera fusca on Coreopsis lanceolata. D Phyllactinia mali on Pyrus pashia. E. Erysiphe betae on Chenopodium sp. F Phyllactinia juglandis on Juglans regia. G Golovinomyces cichoracearum on Abelmoschus esculentus.

\section{Discussion}

Being a north Indian hilly state and part of North Western Himalaya, Himachal Pradesh has faourable environmental conditions for growth and development of powdery mildew fungi. Several studies on these fungi have already been carried out in the state (Chona et al. 1960, Gill et al. 1961, Lall \& Gupta 1965, Sohi \& Nayar 1969 a, b, Singh \& Nagaich 1971, Singh \& Raj 1973, Bhardwaj et al. 1985, Ahmad et al. 2007, Gupta et al. 2016, Gautam 2014 a, b, 2016 a, b, 2017). Paul \& Thakur (2006b) carried out a detailed survey and reported occurrence of the fungi from almost every district of the state.

Although, the number of records from Lahul \& Spiti, Mandi, Kangra, Kullu, Solan and Shumla are higher than from other districts, more genera are found in Solan, Kangra and Mandi. There are districts of the state that have not yet been investigated in any detail. We did not find any published record from district Hamirpur. During the literature survey we came across a study conducted by Braun \& Paul (2009) in which taxonomy and nomenclature of new Indian powdery mildew taxa described by Paul \& Thakur (2006b) were revised and reassessed on the base of the 
current phylogenetic generic concept of the Erysiphaceae. However, molecular studies of powdery mildew fungi are still required besides morphological taxonomy, which will not only help in revision and reassessment of the existing fungal species, but also help to find their correct taxonomic position as well. As such, we hope that the data presented in this paper will provide both a reference work and be an incentive for further work and encourage others to continue adding to the body of information available on the powdery mildews of North Western Himalaya as well as from India.

\section{Acknowledgements}

Authors gratefully thank their respective organizations for encouragement and every possible support to carry out this piece of scientific work.

\section{References}

Ahmad Nasim, Sarbhoy AK, Kamal. 1995 - New powdery mildews from India. Mycological Research 99(3), 374-376.

Ahmad N, Sarbhoy AK, Kamal. 1998 - A new variety and two new species of powdery mildews from India. Mycological Research 102(1), 30-32.

Ahmad N, Sarbhoy AK, Kamal, Agarwal DK. 2006 - Some new powdery mildews from northern India. Indian Phytopathology 59(2), 219-222.

Ahmad N, Agarwal DK, Bambawal OM, Puzari KC. 2007 - Erysiphaceae of India. Monographic Treatment. Venus Printers and Publisher, New Delhi.

Bharat NK. 2003 - Powdery mildew of matricaria in Himachal Pradesh. Plant Disease Research 18, 202.

Bharat NK. 2006. Occurrence of powdery mildew on seabuckthorn in Himachal Pradesh. The Indian Forester 132(4), 517.

Bharat NK. 2009 - Occurrence of perfect stage of Sphaerotheca fuliginea on calendula in dry temperate zone of Himachal Pradesh. Indian Phytopathology 62, 541.

Bharat NK. 2010 - A powdery mildew disease on Selinum veginatum. Indian Forester 136(12), $1717-1718$.

Bharat NK. 2013 - Study on the occurrence of perfect stage of okra powdery mildew in Himachal Pradesh. International Journal of Farm Sciences 3(2), 52-55.

Bharat NK, Bhardwaj LN. 2000 - Occurrence of perfect stage of powdery mildew of apple in north-western Himalayas. Plant Disease Research 15, 81-82.

Bharat NK, Gupta AK. 2010 - Fig (Ficus carica L.) - new host record of Uncinula aspera Doige var. aspera U. Braun. Indian Phytopathology 63(3), 360.

Bhardwaj CL, Paul YS, Singh BM. 1985 - Some new leguminous hosts of powdery mildew. Indian Journal of Mycology and Plant Pathology 15(3), 292.

Bilgrami KS, Jamaluddin, Rizwi MA. 1991 - Fungi of India: list and references. Today and Tomorrow's Printers \& Publishers, New Delhi, India.

Braun U, Cook RTA. 2012 - Taxonomic Manual of the Erysiphales (Powdery Mildews). CBS Biodiversity Series.

Braun U, Paul YS. 2009 - The Indian Erysiphaceae revisited. Nova Hedwigia 89(3-4), 371-395.

Butler EJ, Bisby GR. 1931 - The Fungi of India. Imperial Council of Agricultural Research in India. Science Monograph 1. XVIII.

Chona BL, Kapoor JN, Gill HS. 1960 - Studies on powdery mildew from India. Indian Phytopathology 13, 72-75.

Gautam AK. 2014a - Powdery mildew of Celtis australis: a report from Himachal Pradesh, India. Plant Pathology \& Quarantine 4(1), 14-16.

Gautam AK. 2014b - Studies on some powdery mildew of Himachal Pradesh, India. Australasian Mycologist 32, 10-13. 
Gautam AK, Avasthi S. 2016a - Ampelomyces quisqualis Ces. - A mycoparasite of Euphorbia hirta powdery mildew in Himachal Pradesh, India. Journal of Phytopathology and Pest Management 3(2), 64-70.

Gautam AK, Avasthi S. 2016b - Ampelomyces quisqualis - a remarkable mycoparasite on Xanthium strumarium powdery mildew from Himachal Pradesh India. Journal on New Biological Reports 5(1), 1-6.

Gautam AK, Avasthi S. 2017 - Short communication: some additions to powdery mildews (Erysiphales: Fungi) of Northwestern Himalayas. Nusantara Bioscience 9, 52-56.

Gautam AK, Avasthi S, Bhadauria R. 2012 - New plant fungal diseases during 21st century in India. LAP Lambert Academic Publishing, Saarbrucken, Germany.

Gill HS. 1968 - Studies on powdery mildew from India - III. Mycopathologia et Mycologia Applicata 35, 215-216.

Gill HS, Munjal RL, Chona BL. 1961 - Studies on powdery mildew from India - II. Indian Phytopathology 14, 174-178.

Gupta AK, Bhardwaj LM. 1998 - Additional hosts of Leviellula taurick (Lev.) G. Arnaud from India. Indian Phytopathology 51(1), 104.

Gupta SK, Sharma YP, Sharma J, Warpade S, Gupta M. 2016 - Occurrence of capsicum powdery mildew under protected conditions in Himachal Pradesh. Plant Disease Research 31(2), 169170.

Hosagoudar VB, Agarwal DK. 2009 - Powdery mildews of India: check list. Associated Publications, Delhi, India.

Jamaluddin S, Goswami MG, Ojha BM. 2004 - Fungi of India (1989-2001). Scientific Publishers (India), Jodhpur.

Kala SP, Gaur RD. 1983 - Some new hosts and fungal records from India. Indian Phytopathology $36,370$.

Kumar K, Garg SK, Ahmad ZU. 1975 - Addition to the fungi of India. Journal of Mycology and Plant Pathology 5,107.

Lall G, Gupta JN. 1965 - Notes on powdery mildew from India - III. Indian Phytopathology 18, 210-213.

Mukherji KG, Juneja RC. 1974 - Fungi of India: supplement to the list of Indian Fungi, 19621972. Emkay Publication, Delhi, India.

Pap P, Ranković B, Maširević S. 2013 - Effect of temperature, relative humidity and light on conidial germination of oak powdery mildew (Microsphaera alphitoides Griff. et Maubl.) under controlled conditions. Archives of Biological Sciences 65(3), 1069-1077.

Paul YS. 1984 - Uncinulla ehretiae on Ehretia accuminera. Indian Phytopathology 37, 742.

Paul YS. 1997 - A new species of Phyllactinia from India. Indian Phytopathology 50(2), 291-292.

Paul YS, Bhardwaj CL. 1982 - Reinwardtia indica - a new host record for Oidiopsis taurica. Indian Phytopathology 35, 725.

Paul YS, Bhardwaj LN. 1987 - Powdery mildews on Prinsepia utilis Royle from India. Indian Forester 113(11), 753-754.

Paul YS, Bhardwaj LN. 2001 - New species of powdery mildew fungi from India. Journal of Mycology and Plant Pathology 31(1), 88-89.

Paul YS, Kapoor JN. 1983 - A revision of Erysiphe polygoni sensu Salmon from India. Indian Phytopathology 36, 245-250.

Paul YS, Kapoor JN. 1986 - Taxonomy of Oidiopsis scalia found in India. Kavaka 14(1-2), 61-62.

Paul YS, Kaul JL. 1988 - Powdery mildew of India - New records. Indian Journal of Mycology and Plant Pathology 17(2), 173-174.

Paul YS, Munjal RL. 1982 - New host record for powdery mildew from India. Indian Phytopathology 35, 170-171.

Paul YS, Pal J. 1984 - A taxonomic note on powdery mildew of Galinsoga parviflora. Himachal Journal of Agricultural Research 10(2), 70. 
Paul YS, Thakur V. 2004a - New species of Erysiphaceae from India. Journal of Mycology and Plant Pathology 34(3), 938-940.

Paul YS, Thakur VK. 2004b - Arthrocladiella altheae sp. nov. - An addition to Indian Erysiphaceae. Journal of Mycology and Plant Pathology 34(3), 946-947.

Paul YS, Thakur VK. 2006a - Species of Erysiphaceae described as new from India. Journal of Economic and Taxonomic Botany Additional Series 26, 17-79.

Paul YS, Thakur VK. 2006b - Indian Erysiphaceae. Scientific Publishers, Jodhpur, India.

Pawar VP, Patil VA. 2011 - Occurrence of powdery mildew on some wild plants from Khandesh region of Maharashtra state. Recent Research in Science and Technology 3(5), 94-95.

Sarbhoy AK, Agarwal DK, Varshney JL. 1975 - Fungi of India. Associated Publishing Company New Delhi, India.

Sarbhoy AK, Varshney JL, Agarwal DK. 1980 - Fungi of India. Navyug Traders Publishers, New Delhi, India.

Sharma PN, Kapoor AS, Tyagi PD. 1992 - Kala zira - a new host record of Erysiphe heraclari. Indian Phytopathology 45(4), 474.

Sharma K, Verma S, Gautam HR, Handa A. 2017 - First report of powdery mildew caused by Golovinomyces cichoracearum on dahlia (Dahlia variabilis) from Himachal Pradesh (India). International Journal of Current Microbiology and Applied Sciences 6(6), 1595-1598.

Singh DS, Nagaich BB. 1971 - Powdery mildew of Nicandra physaloides Gaertn.: a new record. Current Science 46, 26.

Singh RA, Raj S. 1973 - Occurrence of a typical powdery mildew on Stephania rotunda Lour. Current Science 42, 69.

Sohi HS, Nayar SK. 1969a - New records of fungi from Himachal Pradesh - I. Indian Phytopathology 22, 410-412.

Sohi HS, Nayar SK. 1969b - New records of fungi from Himachal Pradesh - III. Indian Phytopathology 22, 462-465.

Sohi HS, Sharma SL, Sachdev KB. 1966 - New records of fungi from Himachal Pradesh - I. Indian Phytopathology 19(2), 235-237.

Soni N, Bhardwaj LN, Sharma RC. 1993 - Some new diseases of Heracleum candicans from India. Plant Disease Research 8(1), 68-69.

Sydow H. 1938 - Fungi Himalayensis. Annales Mycologici 36, 437-442.

Vasudeva RS. 1960 - The Fungi of India. Indian Council of Agricultural Research (ICAR), New Delhi, India. 